\title{
Prevalência de infecção pelo "Mycobacterium tuberculosis" entre alunos da Faculdade de Medicina da Universidade Federal do Rio de Janeiro"
}

\author{
VAnia Maria Carneiro da Silva ${ }^{1}$, Jaqueline R. Oliveira ${ }^{2}$, Flávia Matos Silva dos Santos ${ }^{3}$, \\ ClARISSE MoreIRA dE ARAÚJO ${ }^{3}$, AFRÂNIO LINEU KRITSKI ${ }^{4}$
}

\begin{abstract}
Introdução: O Hospital Universitário da Universidade Federal do Rio de Janeiro (HUCFF), na região Sudeste do Brasil, recebe aproximadamente 300 casos de tuberculose por ano e é nesse hospital que os alunos da Faculdade de Medicina exercem suas atividades. Objetivos: Determinar a prevalência de infecção pelo Mycobacterium tuberculosis (Mtb) entre alunos da Faculdade de Medicina da UFRJ.

Desenho do estudo: Estudo transversal entre estudantes de medicina em diferentes níveis de treinamento. Informações sobre características sociodemográficas, vacinação pelo BCG e exposições potenciais à tuberculose foram obtidas através de questionário padronizado. O teste tuberculínico foi usado para determinar a prevalência de infecção pelo Mtb. Resultados: Os estudantes de medicina tiveram prevalência crescente de positividade ao teste tuberculínico de acordo com o progredir de suas atividades práticas $(4,6 \%, 7,8 \%, 16,2 \% ; P=0,002)$. Os riscos foram maiores durante os anos de treinamento clínico, quando os estudantes de medicina tiveram contato mais freqüente com pacientes. Conclusões: Os estudantes de medicina que desenvolvem seu treinamento no HUCFF/UFRJ podem estar sob aumentado risco de se infectar pelo Mycobacterium tuberculosis. Faz-se necessário um programa de realização de teste tuberculínico, de rotina, para confirmação de viragem tuberculínica, combinado com intervenções para reduzir o risco de transmissão nosocomial no local da prática clínica. (J Pneumol 2001;27(2):77-82)
\end{abstract}

\section{Prevalence of mycobacterium tuberculosis infection among medical students of Federal University of Rio de Janeiro}

Introduction: The teaching hospital, HUCFF, Southeastern Brazil, annually receives three hundred tuberculosis cases. Medical students have their practice at that hospital. Objective: To determine the prevalence of Mycobacterium tuberculosis (Mtb) infection among the students of the UFRJ School of Medicine. Design: Cross-sectional study of medical students at different levels of

their training programs. Information about social and demographic characteristics, BCG vaccination history, and potential exposures to tuberculosis were obtained with a standardized questionnaire. Tuberculin skin testing was used to determine the prevalence of tuberculosis infection. Results: The prevalence of tuberculin skin testing positivity among medical students increased as they advanced to higher training levels $(4.6 \%, 7.8 \%, 16.2 \%$, respectively; $P=$ 0.002). The risks were greatest during the years of clinical training, when medical students had more contact with patients. Conclusions: Medical students in the HUCFF/UFRJ may be at an increased risk of $\mathrm{M}$. tuberculosis infection. A program of routine tuberculin skin testing, combined with interventions to confirm TST conversion and to reduce the risk of nosocomial transmission in the workplace is needed.

* Trabalho realizado na Faculdade de Medicina da Universidade Federal do Rio de Janeiro.

1. Professora Assistente; Doutoranda em Clínica Médica pela UFRJ, área de concentração: Pneumologia.

2. Enfermeira do Instituto de Doenças do Tórax.

3. Aluna do curso de graduação, iniciação científica.

4. Professor adjunto de Pneumologia; Coordenador do Programa de Controle de Tuberculose Hospitalar do Hospital Universitário Cle- mentino Fraga Filho; Coordenador da Unidade de Pesquisa em Tuberculose do Serviço de Pneumologia do Hospital Universitário Clementino Fraga Filho.

Endereço para correspondência - Dra. Vania M.C. Silva, Rua Santa Amélia, 88/A/803 - 20260-030 - Rio de Janeiro, RJ, Brasil. E-mail: vaniasilva@alternex.com.br

Recebido para publicação em 5/7/00. Reapresentado em 20/ 9/00. Aprovado, após revisão, em 16/10/00. 
Descritores - Infecção hospitalar. Estudantes de medicina. Fatores de risco. Estudos transversais.

Key words - Nosocomial infection. Medical students. Risk factors. Cross-sectional studies.

\section{INTRODUÇÃO}

Estudantes de medicina da UFRJ são provenientes de famílias com características socioeconômicas muito acima da renda salarial mínima nacional. Em estudo prévio realizado no campus da UFRJ $J^{(1)}$, entre todos os centros, foi verificado que $70 \%$ dos estudantes de medicina são provenientes de famílias com renda superior a dez salários mínimos mensais. Conseqüentemente, esses estudantes poderiam apresentar prevalência de infecção pelo Mycobacterium tuberculosis mais baixa do que a população em geral(2).

O HUCFF atende aproximadamente 300 casos de TB anualmente $e^{(3)}$. Esse hospital universitário é também um centro de referência para AIDS. Os estudantes de medicina têm progressivamente maior contato com pacientes a partir do quinto período da faculdade de medicina, enquanto os alunos do curso básico não participam da assistência médica. A prevalência de alunos infectados no HUCFF e no CCS não era conhecida.

Foi realizado um estudo transversal usando o teste tuberculínico para determinar a prevalência da infecção pelo Mycobacterium tuberculosis entre os alunos de medicina nos diferentes semestres de estudo, comparando alunos do curso básico com os dos semestres intermediários e do internato. Verificamos também as possíveis associações entre a positividade ao TT e os fatores de risco potenciais como vacinação pelo BCG, contato domiciliar com tuberculoso, as atividades dos alunos no CCS, no HUCFF $e$ fora do HUCFF. A presença de associação significante entre TT positivo $e$ atividades dos alunos no hospital universitário sugeriria a necessidade da implementação de medidas de biossegurança $e$ administrativas visando a redução do risco de transmissão nosocomial pelo Mycobacterium tuberculosis.

\section{Casuística e mÉTOdos}

\section{População de estudo}

Foi realizado um estudo transversal com a finalidade de descrever a prevalência da positividade ao TT e os fatores de risco para infecção pelo Mtb entre estudantes de medicina do curso básico (CCS), intermediário e internato (HUCFF) da Faculdade de Medicina da UFRJ, no período de outubro de 1996 a outubro de 1997. Os 1.200 alunos inscritos naquele ano foram candidatos para o estudo. Estudantes com história de tuberculose prévia ou em vigência de terapia imunossupressora foram excluídos. $\mathrm{O}$
Siglas e abreviaturas utilizadas neste trabalho

HUCFF - Hospital Universitário Clementino Fraga Filho

UFRJ - Universidade Federal do Rio de Janeiro

CCS - Centro de Ciências da Saúde

TB - Tuberculose

Mtb - Mycobacterium tuberculosis

PPD - Derivado protéico purificado

BCG - Bacilo de Calmette-Guérin

TST - Tuberculin skin test

TT - Teste tuberculínico

SM - Salário mínimo

$\mathrm{RC}$ - Razão de chances

CRPHF - Centro de Referência Professor Hélio Fraga

CDC - Centers for Disease Control and Prevention

IUATLD - International Union Against Tuberculosis and Lung

Diseases

OMS - Organização Mundial de Saúde

estudo foi aprovado pelo comitê de ética de ambos os centros.

Aproximadamente 200 alunos iniciam o curso médico, a cada ano, na Faculdade de Medicina da UFRJ. Os alunos do curso básico assistem a suas aulas no CCS, onde não há contato com pacientes. No final do segundo ano $e$ início do terceiro (quinto semestre) os alunos passam a ter contato de quatro horas por semana com os pacientes. A partir do quinto semestre até o internato o contato é crescente em número de horas, chegando a oito horas diárias no último ano e meio do curso médico. Os participantes do estudo foram estratificados em três niveis: básico (um a um e meio semestre de estudo na faculdade de medicina), intermediário (quatro a oito semestres de estudo) e internato (nove a 12 semestres de estudo). Os locais de potencial exposição à TB foram definidos como: o contato familiar, o CCS, o HUCFF e outros hospitais onde os alunos realizam estágios.

Os participantes do estudo forneceram consentimento por escrito e completaram um questionário que informava sobre as características sociodemográficas, história de vacinação pelo BCG, tempo de estudo na Faculdade de Medicina, exposição potencial à TB em casa, no CCS, no HUCFF e outros hospitais. A vacinação pelo BCG foi avaliada pela presença da cicatriz vacinal no braço direito.

\section{Técnicas do TT}

O TT foi realizado usando a técnica de Mantoux ${ }^{(4)}$. Foram utilizadas seringas e agulhas (número 27) descartáveis. Foi injetado $0,1 \mathrm{ml}$ de PPD (purified protein derivative, PPD-Rt 23, 2TU) na face volar do antebraço esquerdo de cada aluno. O PPD-Rt23 (State Serum Institute, Dinamarca) foi preparado pelo Centro Nacional de Referência para Tuberculose Professor Hélio Fraga (CRPHF) para ser equivalente ao 5UT-PPD standard. O TT foi aplicado por profissional treinado pelo Ministério da Saúde. 
A região de enduração no sítio de aplicação do TT foi medida pelo método palpatório, 48-72 horas após a injeção. Um TT positivo ou reator foi definido como: uma enduração medindo $\geq 10 \mathrm{~mm}$, um fenômeno booster positivo ou um segundo TT (uma semana mais tarde) $\geq 10 \mathrm{~mm}$. Um fenômeno booster positivo foi definido como positivo se a enduração do segundo TT foi $\geq 10 \mathrm{~mm}$ e mediu, pelo menos, $6 \mathrm{~mm}$ a mais do que a enduração do primeiro TT. Esta classificação visou a comparação com dados da literatura internacional ${ }^{(5,6)}$.

Os alunos com reações $\geq 10 \mathrm{~mm}$ foram considerados positivos ou reatores e não foram retestados. Aqueles com reações $<10 \mathrm{~mm}$ foram retestados uma semana mais tar$\mathrm{de} e^{(4)}$. Neste segundo momento, foi realizado um painel cutâneo (testes de anergia) para distinguir um resultado de um TT negativo de anergia. Toxóide tetânico (diluído 1:5 em albumina humana) e candidina (diluição de 1:100 de uma cultura estéril de filtrados de Candida albicans) foram preparados e cedidos pelo serviço de Imunologia do HUCFF/UFRJ, sendo este material o mesmo que é usado rotineiramente pelo serviço, para avaliação dos seus pacientes. Os testes cutâneos foram aplicados via intradérmica na inserção do músculo deltóide. A leitura foi realizada 48-72h mais tarde. Uma enduração de $\geq 2 \mathrm{~mm}$ foi considerada evidência de hipersensibilidade do tipo tardio em qualquer dos três testes realizados (PPD, candidina ou toxóide) $)^{(7)}$.

\section{TT positivo e tuberculose ativa}

Todos os estudantes que apresentaram positividade ao TT foram examinados por um pneumologista, que solicitou telerradiografia de tórax e baciloscopia de escarro se as imagens radiológicas fossem típicas de TB.

\section{Análise dos dados}

Os dados oriundos dos questionários e os resultados do TT foram coletados, armazenados e analisados usando o programa Epi-Info 6.04 (Centers for Disease Control and Prevention, CDC, Atlanta, Georgia). Análises adicionais foram realizadas usando o programa Intercooled Stata 5.0 (Stata Corporation, College Station, Texas). Foi usado o teste do $\chi^{2}$ ou o teste exato de Fisher para testar as associações entre variáveis categóricas independentes e o desfecho principal, a positividade ao TT. Algumas variáveis contínuas foram categorizadas. Foi usado o teste do $\chi^{2}$ para tendência linear para testar associação da variável ordinal, nível de estudo, com o desfecho. A razão de chances e o intervalo de confiança de 95\% (95\% IC) foram computados tanto para análise univariada como para análise multivariada. Uma análise de regressão logística foi realizada para identificar os fatores independentes relacionados ao TT positivo na população de estudo. No modelo multivariado entraram as variáveis que obtiveram valor de $P \leq 0,05$.

\section{RESUlTADOS}

Dos 1.200 estudantes de medicina elegiveis para o estudo, 854 foram contactados e convidados para participar do estudo. Os demais estudantes não foram encontrados nos endereços residenciais disponíveis na secretaria da faculdade, ou por telefone, ou ainda nas atividades que supostamente deveriam estar desenvolvendo. Entre os contactados, 545 alunos (545/854, ou 63,8\%) entraram no estudo e responderam a um questionário seguido do TT. Entre os 545 participantes, 90 (16,5\%) não completaram todas as partes do estudo: $30(5,5 \%)$ não completaram o questionário, dois $(0,4 \%)$ foram excluídos devido a TB prévia, e $58(10,6 \%)$ não retornaram para leitura do teste. Trezentos e doze dos 455 estudantes de medicina $(68,6 \%)$ completaram o protocolo do TT em dois tempos (Tabela 1).

TABELA 1

Características dos participantes do estudo, Rio de Janeiro, 1996-1997

\begin{tabular}{|c|c|c|}
\hline \multirow[b]{2}{*}{ Características } & \multicolumn{2}{|c|}{ Estudantes de medicina } \\
\hline & Número/Total & $(\%)$ \\
\hline $\begin{array}{l}\text { Número de estudantes que } \\
\text { completaram pelo menos um TT }\end{array}$ & $455 / 545$ & $(83,5)$ \\
\hline $\begin{array}{l}\text { Número de estudantes que } \\
\text { completaram o TT em dois tempos }\end{array}$ & $312 / 455$ & $(68,6)$ \\
\hline Média de idade (anos) \pm DP & $21,3 \pm 2,3$ & \\
\hline Gênero feminino & $246 / 455$ & $(54,1)$ \\
\hline Gênero masculino & $209 / 455$ & $(45,9)$ \\
\hline $\begin{array}{l}\text { Participantes com história } \\
\text { de vacinação pelo BCG }\end{array}$ & $436 / 455$ & $(83,3)$ \\
\hline $\begin{array}{l}\text { Participantes com história de } \\
\text { vacinação e revacinados pelo BCG* }\end{array}$ & $138 / 146$ & $(94,5)$ \\
\hline Revacinados há cinco anos & $8 / 146$ & $(5,5)$ \\
\hline Cicatriz vacinal (BCG) presente & $297 / 439$ & $(67,6)$ \\
\hline $\begin{aligned} \text { Renda familiar } & <10 \text { SM ao mês } \\
& \geq 10 \text { SM ao mês }\end{aligned}$ & $\begin{array}{r}30 / 447 \\
418 / 447\end{array}$ & $\begin{array}{r}(6,7) \\
(93,5)\end{array}$ \\
\hline Contato domiciliar com tuberculose & $34 / 455$ & $(7,4)$ \\
\hline $\begin{array}{l}\text { Estágio fora do HUCFF } \\
\text { Sem estágio fora do HUCFF }\end{array}$ & $\begin{array}{l}139 / 455 \\
316 / 455\end{array}$ & $\begin{array}{l}(30,5) \\
(69,4)\end{array}$ \\
\hline $\begin{array}{l}\text { Nível básico (períodos) } \\
\text { Nível intermediário (períodos) } \\
\text { Nível internos (períodos) }\end{array}$ & $\begin{array}{l}152 / 455 \\
167 / 455 \\
136 / 455\end{array}$ & $\begin{array}{l}(33,4) \\
(36,7) \\
(29,9)\end{array}$ \\
\hline
\end{tabular}


Entre os 455 estudantes de medicina, 33 (7,3\%) reagiram como positivos e não foram retestados. Dos 422 $(92,7 \%)$ que foram inicialmente negativos, todos $(100 \%)$ foram retestados com TT; entretanto, 110 (26\%) não se apresentaram para a leitura. Entre os 312 (73,9\%) alunos que foram retestados e retornaram para a leitura, quatro $(1,3 \%)$ apresentaram o fenômeno de booster e cinco $(1,6 \%)$, enduração $\geq 10 \mathrm{~mm}$ na segunda dose. A positividade total ao TT foi de $9,2 \%(42 / 455)$. A preva- lência de positividade do TT foi de 4,6\% (7/152), 7,8\% (13/167), 16,2\% (22/136), nos niveis básico, intermediário e internato, respectivamente, $P=0,002$. A idade dos participantes, a vacinação com o BCG, averiguada pela presença de cicatriz vacinal, a renda familiar, o gênero, o contato com tuberculoso na família e a realização de estágio fora do HUCFF não estiveram significantemente associados com o TT positivo (Tabelas 2 e 3). Entretanto, os estudantes de medicina de semestres mais avança-

TABELA 2

Resultados da análise univariada dos fatores extra-atividades estudantis associados com um TT positivo entre estudantes de medicina, Rio de Janeiro, 1996-1997

\begin{tabular}{|c|c|c|c|c|c|c|c|}
\hline \multirow{2}{*}{$\begin{array}{c}\text { Fatores extra-atividades } \\
\text { estudantis }\end{array}$} & \multicolumn{2}{|c|}{ TT positivo } & \multicolumn{2}{|c|}{ TT negativo } & \multirow[t]{2}{*}{ RC } & \multirow[t]{2}{*}{ IC } & \multirow[t]{2}{*}{$\boldsymbol{P}$} \\
\hline & $\mathbf{N}$ & $(\%)$ & $\mathbf{N}$ & $(\%)$ & & & \\
\hline & 42 & $(9,2)$ & 413 & $(90,8)$ & & $(95 \%$ IC) & Valor \\
\hline \multicolumn{8}{|l|}{ Idade } \\
\hline$\geq 21$ anos & 26 & $(61,9)$ & 192 & $(46,5)$ & 1,8 & $(0,9-3,7)$ & $0,081^{*}$ \\
\hline$<21$ anos & 16 & $(38,1)$ & 221 & $(53,5)$ & & & \\
\hline Cicatriz BCG presente & 31 & $(73,8)$ & 266 & $(64,4)$ & 1,3 & $(0,6-3,0)$ & $0,469^{*}$ \\
\hline Sem cicatriz de BCG & 11 & $(26,2)$ & 131 & $(31,7)$ & & & \\
\hline Desconhecido & 0 & & 16 & $(3,9)$ & & & \\
\hline \multicolumn{8}{|l|}{ Renda familiar } \\
\hline$<10 \mathrm{SM}$ & 0 & $(97,6)$ & 28 & $(6,8)$ & indef. & $(0,0-1,6)$ & $0,009 *$ \\
\hline$\geq 10 \mathrm{SM}$ & 41 & $(97,6)$ & 378 & $(91,5)$ & & & \\
\hline sem resposta & 1 & $(2,3)$ & 7 & $(1,7)$ & & & \\
\hline Contato familiar com TB & 5 & $(11,9)$ & 29 & $(7,0)$ & 1,1 & $(0,2-5,6)$ & $1,000^{* *}$ \\
\hline Sem contato com TB & 4 & $(9,5)$ & 32 & $(7,0)$ & & & \\
\hline Sem resposta & 28 & $(66,6)$ & $(357)$ & $(86,4)$ & & & \\
\hline
\end{tabular}

TABELA 3

Resultados da análise univariada das atividades estudantis associadas com TT positivo entre estudantes de medicina, Rio de Janeiro, 1996-1997

\begin{tabular}{|c|c|c|c|c|c|c|c|}
\hline \multirow[t]{2}{*}{ Atividades estudantis } & \multicolumn{2}{|c|}{ TT positivo } & \multicolumn{2}{|c|}{ TT negativo } & \multirow[t]{2}{*}{$\mathbf{R C}$} & \multirow[t]{2}{*}{ IC } & \multirow[t]{2}{*}{$\boldsymbol{P}^{*}$} \\
\hline & $\mathbf{N}$ & $(\%)$ & $\mathbf{N}$ & $(\%)$ & & & \\
\hline & 42 & $(9,2)$ & 413 & $(90,8)$ & & $(95 \%$ IC $)$ & Valor \\
\hline \multicolumn{8}{|l|}{ Local de estudo } \\
\hline HUCFF & 35 & $(83,3)$ & 268 & $(64,9)$ & 2,7 & $(1,1-6,8)$ & 0,024 \\
\hline CCS & 7 & $(16,6)$ & 145 & $(35,1)$ & & & \\
\hline \multicolumn{8}{|l|}{ Nível de estudo } \\
\hline Internato & 22 & $(52,4)$ & 114 & $(27,6)$ & 2,8 & $(1,4-5,7)$ & 0,001 \\
\hline Básico e intermediário & 20 & $(47,6)$ & 299 & $(72,4)$ & & & \\
\hline Estágio fora do HUCFF & 17 & $(40,5)$ & 122 & $(29,5)$ & 1,6 & $(0,8-3,2)$ & 0,196 \\
\hline Sem estágio fora do HUCFF & 25 & $(59,5)$ & 291 & $(70,5)$ & & & \\
\hline
\end{tabular}

* teste do $\chi^{2} ; \mathrm{TT}=$ teste tuberculínico; RC = razão de chances; IC = intervalo de confiança; HUCFF = Hospital Universitário Clementino Fraga Filho; $\mathrm{CCS}=$ Centro de Ciências da Saúde; Internato = 9-12 semestres de estudo; Básico e intermediário = 1-8 semestres de estudo. 
dos (internos) tiveram maior chance de apresentar TT positivo comparados com alunos do curso básico e intermediário ( $\mathrm{RC}=2,8 ; 95 \% \mathrm{IC}=1,4-5,7 ; P=0,001)$ (Tabela 3). A localização onde os alunos realizavam as atividades práticas, tais como o CCS e o HUCFF, também esteve significantemente associada com TT positivo $(P=0,024$ (Tabela 3).

O fenômeno booster não foi associado com gênero $(P$ $=0,627)$, idade $(P=1,000)$, vacinação pelo BCG $(P=$ $0,611)$, ou semestre de estudo $(P=0,575)$.

$\mathrm{Na}$ análise multivariada, controlando para potenciais variáveis de confusão como idade, nível de estudo (períodos) e localização do aluno, o único fator que se mostrou como independente para TT positivo foi o nivel de estudo do aluno ( $\mathrm{RC}=2,94$; IC 95\% $=1,13-7,59 ; P=0,002$ ). Entre os 42 alunos que tiveram TT positivo, $37(88,1 \%)$ se apresentaram com telerradiografia de tórax normal. Três alunos não realizaram radiografia. Quatro $(1,3 \%)$ alunos entre os 312 que realizaram o painel cutâneo mostraram-se anérgicos.

\section{DISCUSSÃO}

Nosso estudo apresentou limitações. Nem todos os alunos elegiveis para o estudo participaram do mesmo. Do total de 1.200 inscritos na Faculdade de Medicina no ano do estudo, apenas $73 \%$ puderam ser contactados. O tamanho amostral para um inquérito nesta população, para prevalência de $25 \%$ de infectados, seria de 232 alunos. Embora tenhamos excedido esse número, um viés de seleção pode ter ocorrido, na medida em que estudantes que já sabiam ser reatores ao TT podem não ter participado, ou, aqueles que se achavam sob maior risco de ser infectados podem ter participado mais ativamente. Neste estudo, 9,2\% dos estudantes de medicina foram positivos quando realizaram o TT em um ou dois tempos. Aqueles estudantes provavelmente foram infectados pelo Mycobacterium tuberculosis, muito embora haja uma chance de eles terem sido infectados por outras micobactérias. A prevalência de infecção pelo Mtb estimada para a população em geral nas Américas (exceto Estados Unidos da América do Norte e Canadá) é de $25 \%^{(2)}$. Atribuímos nossos resultados inferiores à faixa etária jovem estudada $e$ ao nível socioeconômico destes alunos, muito superior ao da população em geral.

Os estudos transversais são limitados na capacidade de indicar associações causais. Um estudo longitudinal seria mais apropriado para avaliação de risco de infecção, taxa de conversão e fatores associados ao risco de infecção. Entretanto, através dos resultados obtidos, com a crescente tendência de haver maior proporção de infectados, no decorrer dos períodos, levantamos a hipótese de estar ocorrendo viragem tuberculínica neste tempo de práticas clínicas mais freqüentes. Ferreira( ${ }^{(8)}$ mostrou prevalência de $20 \%$ de infectados pelo Mtb entre alunos de medicina, em estudo realizado no Hospital Universitário Gafreé Guinle, na cidade do Rio de Janeiro, para uma faixa etária semelhante à nossa, e uma diferença significante entre os períodos de internato e curso básico. Nossa amostra de alunos não infectados (413/455), assim como a de Ferreira $^{(8)}(355 / 446)$, indica uma proporção relativamente elevada de alunos ainda não expostos ao Mtb exercendo atividades em hospitais universitários, ambos centros de referência para AIDS na cidade do Rio de Janeiro.

A prevalência mais baixa de infectados no nosso estudo, comparada com a do de Ferreira ${ }^{(8)}$, pode ser explicada pela adoção de isolamento respiratório( ${ }^{(9)}$ no nosso hospital, medida que não ocorre no hospital onde foi realizado o estudo do referido autor. Um estudo recente realizado no $\mathrm{HUCFF}^{(10)}$ descreveu prevalência de $51 \%$ de profissionais de saúde infectados pelo Mtb. Esta diferença para com o nosso estudo pode ser explicada pela idade dos participantes (habitual variável de confusão neste tipo de estudo), pelas diferentes e mais freqüentes exposições ao Mtb neste último grupo e pelas diferenças socioeconômicas. Muñoz-Barret et al. ${ }^{(11)}$ descreveram prevalência de $41 \%$ de infectados pelo Mtb entre estudantes de medicina, de quinto ano médico, e de $14 \%$ entre alunos do curso básico, no México.

Através dos dados mencionados, dentro de um hospital universitário, o aluno de graduação poderia apresentar-se como um marcador adicional da transmissão nosocomial pelo Mtb se forem adotadas medidas de biossegurança.

Nossos resultados mostraram falta de associação entre a positividade ao TT $e$ a vacinação com BCG. Este achado contradiz estudos na literatura ${ }^{(11,12)}$. A maioria dos participantes do nosso estudo foi vacinada quando criança. No nosso trabalho, a positividade ao TT provavelmente não deve ser devida à vacinação pelo BCG. Assim, o TT continua útil na avaliação de infecção nosocomial por Mtb nesta população. O fenômeno booster também não esteve associado com a vacinação pelo BCG. Outros estudos serão necessários para abordar o referido fenômeno, principalmente em populações de elevada prevalência de micobactéria atípica na região e de vacinação com BCG recente.

Devido à magnitude da tuberculose no Brasil, a maioria dos brasileiros é considerada infectada quando adulto jovem. Dessa forma, o teste tuberculínico não é correntemente aplicado nas escolas médicas. Estudos semelhantes deveriam ser conduzidos em outras escolas médicas assim como em outras faculdades no Brasil. Um estudo longitudinal nos informará quanto ao risco de infecção nosocomial entre alunos de escolas médicas. De acordo com nossos resultados, recomendamos pelo menos um 
teste tuberculínico anual entre alunos de medicina que forem negativos ao teste tuberculínico no momento de ingresso na faculdade, assim como quimioprofilaxia quando houver viragem tuberculínica. Esta recomendação é consistente com o manual dos CDC, IUATLD e OMS ${ }^{(13,14)}$.

\section{REFERÊNCIAS}

1. Silva VMC, Mesquita ARM, Vieira IMM, Pantoja AV, Adriano HCP. Tabagismo entre estudantes da UFRJ. XVIII Jornada de Iniciação Científica da UFRJ, Rio de Janeiro, 1995 (resumo 357).

2. Sudre P, ten Dam G, Kochi A. Tuberculosis: a global overview of the situation today. Bull World Health Organ 1992;70:149-159.

3. Kritski AL. Tuberculosis and HIV: renewed challenge. Mem Inst Oswaldo Cruz 1998;3:417-421.

4. Centers for Disease Control and Prevention. Guidelines for preventing the transmission of Mycobacterium tuberculosis in health-care facilities. MMWR Morb Mortal Wkly Rep 1994;43(RR-15):1-104.

5. Thompson NJ, Glassroth J, Snider D, Farer L. The booster phenomenon in serial tuberculin testing. Am Rev Respir Dis 1979;119:587597.

6. Bass JB, Serio RA. The use of repeated skin tests to eliminate the booster phenomenon in serial tuberculin skin testing. Am Rev Respir Dis 1981;123:394-396.

7. Centers for Disease Control and Prevention. Purified protein derivative PPD tuberculin anergy and HIV infection: guidelines for anergy testing and management of anergic persons at risk of tuberculosis. MMWR Morb Mortal Wkly Rep1991;40(RR-5):27-33.

8. Ferreira, FM. Prevalência de infecção por Mycobacterium tuberculosis em estudantes de medicina da Universidade do Rio de Janeiro UNIRIO [Tese de Mestrado]. Rio de Janeiro: UFRJ, 1998.

9. Bangsberg DR, Crowley K, Moss A, Dobkin JF, McGregor C, Neu HC. Reduction in tuberculin skin test conversion among medical house staff associated with improved tuberculosis infection control practices. Infect Control Hosp Epidemiol 1997;18:566-570.

10. Muzzy de Souza, GR Tuberculose em profissionais em hospital geral: análise do efeito "booster" e conversão do teste tuberculínico [Tese de Doutorado] Rio de Janeiro: Universidade Federal do Rio de Janeiro, 2000.

11. Muñoz-Barret JM, Macias-Hernandez AE, Hernandez-Ramos I. Comparative tuberculin reactivity to two protein derivatives. Rev Invest Clin 1996;48:377-381.

12. Schwartzman K, Loo V, Pasztor J, Menzies D. Tuberculosis infection among health care workers in Montreal. Am J Respir Crit Care Med 1996; 154:1006-1012.

13. A joint statement of the International Union Against Tuberculosis and Lung Disease (IUATLD) and the Tuberculosis Programme of the World Health Organization (WHO). Control of tuberculosis transmission in health care settings. Tuberc Lung Dis 1994;75:94-95.

14. Harries A D, Maher D, Nunn P. Practical and affordable measures for the protection of health care workers from tuberculosis in low-income countries. Bull World Health Organ 1997;75:477-489. 